\title{
Cuboctahedric Higgs oscillator from the rational Calogero model
}

\author{
Tigran Hakobyan,, , , 丹 Armen Nersessian,, , , 丹 and Vahagn Yeghikyan ${ }^{1}$ \\ ${ }^{1}$ Yerevan State University, 1 Alex Manoogian, 375025 Yerevan, Armenia \\ ${ }^{2}$ Yerevan Physics Institute, 2 Alikhanyan Br., 375036 Yerevan, Armenia \\ ${ }^{3}$ Artsakh State University, 3 Mkhitar Gosh, Stepanakert, Armenia
}

\begin{abstract}
We exclude the center of mass of the $N$-particle rational Calogero model and consider the angular part of the resulting Hamiltonian. We show that it describes the motion of the particle on $(N-2)$ dimensional sphere interacting with $N(N-1) / 2$ force centers with Higgs oscillator potential. In the case of four-particle system these force centers define the vertexes of an Archimedean solid called cuboctahedron.
\end{abstract}

\section{INTRODUCTION}

The Calogero model [1, 2, 3] and its various extensions and generalizations play a distinguished role among other multi-particle integrable systems. They have attracted much attention due to their rich internal structure and numerous applications in many areas of physics (see, e.g., the recent review [4] and references therein).

In the continuum or thermodynamic limit, i.e. for large particle numbers, the Calogero model gives rise to a YangMills theory [5] on a cylinder, while its superconformal extension describes a black hole in the near-horizon limit [6]. In this limit, the system have soliton solutions corresponding to the fundamental excitations [7].

The quantum Calogero model describes free particles with fractional statistics whose type is determined by the interaction strength 8]. Moreover, the variational ground state of the fractional quantum Hall effect (known as the Laughlin state [9]) can be considered as some deformation of the ground state of Calogero model [10]. The trigonometric analogue of the model [1] is related to integrable spin-1/2 chains with long range interactions, which possess a resonating-valence-bond ground state [12]. Recently, the relation to the Benjamin-Ono equation arising in the hydrodynamics of stratified fluids has been established [13].

The Calogero model and its modifications appear also in matrix models [14], $W_{\infty}$-algebras [15], Yangian quantum groups [16], random matrices [17] and many other areas of physics and mathematics.

In this article, we will study the classical rational Calogero system without confining potential. It describes onedimensional particles with inverse-square interaction [1, 2, 3]:

$$
\mathcal{H}=\frac{1}{2} \sum_{i=1}^{N} p_{i}^{2}+\sum_{i<j} \frac{g}{\left(x_{i}-x_{j}\right)^{2}}, \quad\left\{p_{i}, x_{j}\right\}=\delta_{i j} .
$$

One of the important features of the system is its manifest conformal invariance, which was essential for the invention of the model, as well as for its further studies.

In the pioneering paper [1], the three-particle model had been considered first. After excluding the center of mass (with á priory conserving momentum) and taking into account the conformal invariance, the model was reduced to a one-dimensional exactly solvable system on circle considered by Jacobi in the middle of XIX century [18]:

$$
\mathcal{I}=\frac{p_{\varphi}^{2}}{2}+\frac{9 g}{2 \cos ^{2} 3 \varphi} .
$$

For more particles, the analysis of the Calogero model becomes more complicated. In particular, the construction of the complete set of the constants of motion assumes the use of the powerful method of Lax pair [3]. This approach allowed to relate the Calogero system to $A_{N-1}$ Lie algebras, as well as to construct its integrable modifications related to other Lie algebras [19]. The Calogero systems can be obtained from the free-particle system by an appropriate reduction procedure known as the projection method [20]. Recently, it has been generalized to the Calogero model extensions corresponding to the root systems [21].

However, the analog of the system (2) has not been properly studied for the case of more than three particles. Such a study would be an interesting problem from few viewpoints.

\footnotetext{
*Electronic address: hakob@yerphi.am

†Electronic address: arnerses@yerphi.am
} 
Already in the pioneering papers [1, 2] it was observed that the spectrum of the Calogero model with additional oscillator potential is similar to the spectrum of free $N$-dimensional oscillator. It was claimed there that a similarity transformation to the free-oscillator system may exist, at least, in the part of Hilbert space. However, this transformation has been written explicitly only three decades later [22]. In Ref. 23, it has been related to the conformal group $S U(1,1)$. This similarity transformation has a very transparent geometric explanation for the two-particle Calogero model (the "conformal mechanics"): it corresponds to the inversion in the Klein model of the Lobachevsky space, which describes the phase space of the system. A natural way to extend this picture to the multi-particle Calogero system is to identify the coordinates of its "radial" part with the coordinates of the Klein model. In other words, one must extract and investigate the angular part of the system.

Another motivation is connected with the superconformal extensions of Calogero model. In Ref. 23, the authors suggested to use the aforementioned similarity transformation for the construction of $\mathcal{N}=4$ superconformal Calogero system [25]. However, the suggested algebraic scheme is quite non-trivial, and for the cases of four and more particles it assumes the solving of WDVV and other partial differential equations. On the other hand, in Ref. 27 the superconformal extension of the three-particle Calogero model was constructed in a transparent way within the superfield approach by extracting the model (2) from the initial system. This approach seems to be applicable to any-particle Calogero system, under assumption that its angular part admits $\mathcal{N}=4$ supersymmetric extension.

The other stimulation for the study of the angular part of the Calogero model the translation of the discrete symmetries of the one-dimensional multi-particle system to the higher-dimensional one-particle one. This would provide us with á priori integrable higher-dimensional one-particle system with some discrete symmetry.

The purpose of the current article is the investigation of the angular part of the $N$-particle Calogero model with the excluded center of mass. Like in the three-particle case, it is a constant of motion. Hence, its Poisson brackets with the Liouville constants of motions can generate the additional constants of motions. This can give a simple explanation of the superintegrability of Calogero model observed many years ago by Wojciechowski [28]. We show that the angular part itself describes a particle on the $(N-2)$-dimensional sphere, which interacts with the $N(N-1) / 2$ force centers by the Higgs oscillator low. Briefly speaking, we have a $N(N-1) / 2$-center $(N-2)$-dimensional Higgs oscillator. For the $N=4$ case corresponding to particle motion on two-dimensional sphere, the force centers are located at the vertexes of the Archimedean solid cuboctahedron. This observation opens few horizons for further study of the Calogero model. In particular, the investigation of the angular part of the spin-Calogero model and/or its supersymetric extentions becomes especially important due to the possibility of applications in the solid state physics.

The paper is organized as follows.

In Section 2 we exclude the center of mass from the $N$-particle Calogero model and obtain a $(N-1)$-dimensional system characterized by $N(N-1) / 2$ unit vectors. These vectors correspond to the positive roots of the Lie algebra $A_{N-1}$. Then we show that the angular part of the reduced system describes a particle on $(N-2)$-dimensional sphere interacting with the vertexes of the aforementioned vectors by the Higgs oscillator low.

In Section 3 we illustrate the general analysis of Section 2 for the simplest nontrivial case of three-particle Calogero model. The corresponding angular part is given by (2). We show that its Poisson bracket with the third-order Liouville constant of motion coincides with the additional constant of motion. Then we derive an explicit functional dependence between four constants of motion (including the Hamiltonian).

In Section 4 we consider the four-particle system. We show that its angular part describes a multicenter Higgs oscillator on two-dimensional sphere with force centers located at the vertexes of the cuboctahedron.

In the last section we summarize the results and discuss the possible extension to the supersymmetric Calogero systems.

\section{CENTER-OF-MASS SYSTEM}

In order to decouple the center of mass, we introduce a new coordinate system for $N$-particle Calogero model.

$$
y_{0}=\frac{1}{\sqrt{N}} \sum_{i=1}^{N} x_{i}, \quad y_{k}=\frac{1}{\sqrt{N-k+1}}\left(\sqrt{N-k} x_{k}-\frac{1}{\sqrt{N-k}} \sum_{i=k+1}^{N} x_{i}\right), \quad 1 \leq k \leq N-1
$$

Here $y_{0}$ describes the center-of-mass movement, while the remaining $y_{k}$ describe the motion in the certer-of-mass system. The transformation (3) is chosen to be orthogonal:

$$
\sum_{k=1}^{N}\left(d x_{k}\right)^{2}=\left(d y_{0}\right)^{2}+\sum_{k=1}^{N-1}\left(d y_{k}\right)^{2}
$$


Hence, the inverse transformation $x_{k}=\sum_{n=1}^{N} A_{k n} y_{n}$ coincides with its transpose:

$$
A_{k m}= \begin{cases}1 / \sqrt{N} & \text { for } m=0 \\ -1 / \sqrt{(N-m+1)(N-m)} & \text { for } k>m \geq 1 \\ \sqrt{N-k} / \sqrt{N-k+1} & \text { for } m=k \\ 0 & \text { for other } m\end{cases}
$$

Using these formulae, we rewrite the Hamiltonian of the Calogero model in terms of the center-of-mass variables:

$$
\mathcal{H}=\frac{1}{2} \sum_{i=1}^{N} p_{i}^{2}+\sum_{i<j} \frac{g}{\left(x_{i}-x_{j}\right)^{2}}=\frac{p_{0}^{2}}{2}+\tilde{\mathcal{H}}
$$

Here $p_{0}=\sum_{i=1}^{N} p_{i}$ is the conserved total momentum of the $N$-particle Calogero system. The last term is given by the expression

$$
\tilde{\mathcal{H}}=\frac{1}{2} \sum_{i=1}^{N-1} p_{i}^{2}+\sum_{a=1}^{N(N-1) / 2} \frac{g}{2\left(\sum_{k=1}^{N-1} b_{k}^{a} y_{k}\right)^{2}}, \quad\left\{p_{i}, y_{j}\right\}=\delta_{i j}
$$

where $a \equiv(i, j)$ enumerates the pairs of interacting particles, $p_{i}$ (we keep the old notation for them) are the new momenta conjugated to $y_{i}$, and

$$
b_{k}^{a}=b_{k}^{i j}=\frac{A_{i k}-A_{j k}}{\sqrt{2}} .
$$

It is clear that the Hamiltonian with the excluded center of mass $\tilde{\mathcal{H}}$ is a constant of motion of the original system (1). From the orthogonality of the matrix $A_{i k}$ we have:

$$
\sum_{k}\left(b_{k}^{i j}\right)^{2}=1, \quad \cos \alpha_{i j, i^{\prime} j^{\prime}}=\sum_{k} b_{k}^{i j} b_{k}^{i^{\prime} j^{\prime}}=\frac{1}{2} \sum_{k}\left(A_{i k}-A_{j k}\right)\left(A_{i^{\prime} k}-A_{j^{\prime} k}\right)=\frac{1}{2}\left(\delta_{i i^{\prime}}+\delta_{j j^{\prime}}-\delta_{i j^{\prime}}-\delta_{i^{\prime} j}\right) .
$$

So, the coordinates $\mathbf{b}^{a}=\left(b_{1}^{a}, \ldots, b_{N-1}^{a}\right)$ define unit vectors in $(N-1)$-dimensional space, while $\alpha_{i j, i^{\prime} j^{\prime}}$ are the angles between them. In fact, $\mathbf{b}^{a}$ correspond to the positive roots of the Lie algebra $A_{N-1}$ (rescaled by the factor $1 / \sqrt{2}$ ). Indeed, the potential of original model (11) can be presented as the inverse-square sum over all positive roots $\Delta_{+}$of $A_{N-1}$ multiplied by the particle coordinates: $\sum_{\alpha \in \Delta_{+}} g /(\alpha \cdot \mathbf{x})^{2}$ [20]. The orthogonal transformation (3) acts on those roots by mapping them onto $\mathbf{b}^{a}$. Therefore, the last vectors define the same root system.

The reduced system (4), (5) can be interpreted as a one-particle system in $(N-1)$-dimensional space. Let us extract the radius $r$ of the obtained system. This can be done, for instance, in hyperspherical coordinates, where the Hamiltonian (4) takes the following form:

$$
\tilde{\mathcal{H}}=\frac{p_{r}^{2}}{2}+\frac{\mathcal{I}\left(p_{\varphi_{\alpha}}, \varphi_{\alpha}\right)}{r^{2}}, \quad \mathcal{I}\left(p_{\varphi_{\alpha}}, \varphi_{\alpha}\right)=\frac{K_{\mathrm{sph}}\left(p_{\varphi_{\alpha}}, \varphi_{\alpha}\right)}{2}+\sum_{a} \frac{g}{2 \cos ^{2} \theta_{a}}, \quad\left\{p_{\varphi_{\alpha}}, \varphi_{\alpha}\right\}=\delta_{\alpha \beta},
$$

where $\alpha, \beta=2, \ldots, N-1$. Here $K_{\mathrm{sph}}$ is the standard kinetic term of the particle on the hypersphere $S^{N-2}$ with unit radius, $\theta_{a}$ is the angle between $\mathbf{b}_{a}$ and the unit vector $\mathbf{n}=\mathbf{r} / r$ directed from the hypersphere center to the particle.

Since $\mathcal{I}$ is independent from the radial coordinates $p_{r}$ and $r$, it commutes with the Hamiltonian $\tilde{\mathcal{H}}$. So, it is a constant of motion of the Calogero model. Note that this integral is quadratic on the momenta (while in the standard Lax pair approach, the only constant of motion, which is quadratic on momenta, is the Hamiltonian). It is easy to verify that any other integral being in involution with $\mathcal{I}$ must depend on the radial coordinated through the Hamiltonian $\tilde{\mathcal{H}}$. Therefore, it is not in involution, at least, with the integrals having an odd order on $p_{r}$ and is functionally independent from the $N$ commuting integrals constructed using the Lax pair. The matter is that the Calogero model is not only an integrable in the Liouville sense (which means the existence of $N$ integrals being in involution) but is maximally superintegrable with $2 N-1$ functionally independent integrals [28] (see also [29]). Clearly, $\mathcal{I}$ is a function of this complete set of the constants of motion. Moreover, its Poisson bracket action on the Liouville constants of motions generates the additional constants of motion, which are responsible for the superintegrability.

It can be considered as the Hamiltonian of a particle moving on the $(N-2)$-dimensional sphere with $N(N-1) / 2$ force centers defined by the vectors $\mathbf{b}^{a}$. Since this system is invariant under reflections $\mathbf{b}^{a} \rightarrow-\mathbf{b}^{a}$ for any $a$, sometimes 


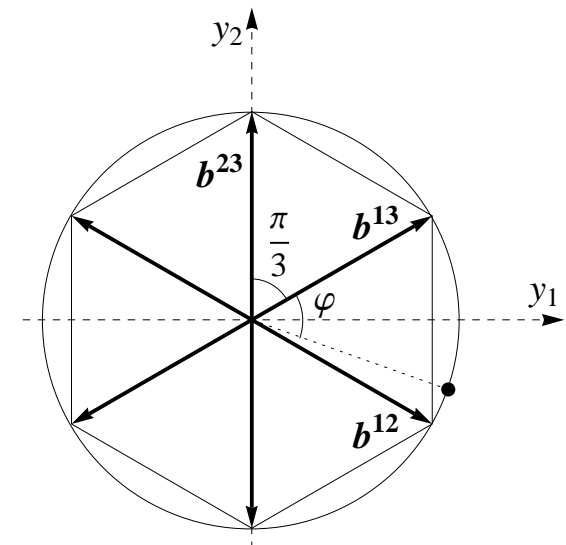

FIG. 1: The force centers $\left(\mathbf{b}^{12}, \mathbf{b}^{23}, \mathbf{b}^{13}\right.$ and their opposites), which form the root system of $s u(3)$ and constitute an hexagon. The angle $\varphi$ describes the position of a particle on cycle.

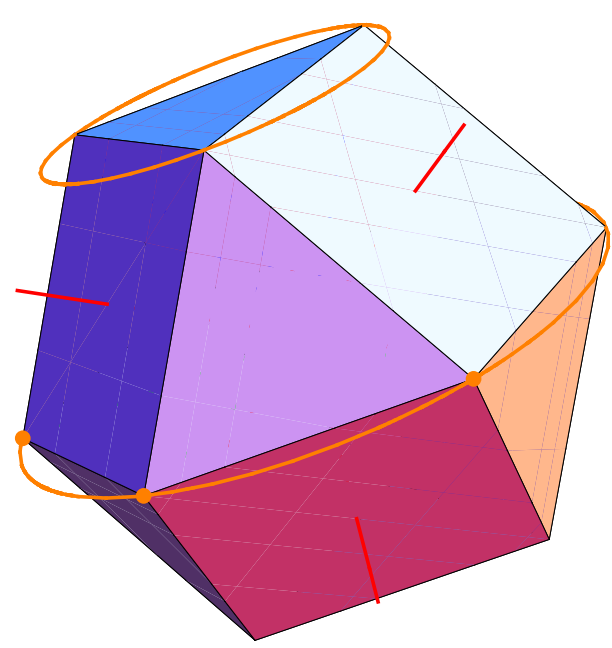

FIG. 2: The vectors (13) together with their opposites form a cuboctahedron and are equivalent to the root system of $s u(4)$. The bold points on the large cycle correspond to $\mathbf{b}^{23}, \mathbf{b}^{34}$ and $\mathbf{b}^{24}$ while the small cycle contains the vertexes of the remaining three vectors. The bold lines are the axes of the coordinate system (15).

it is reasonable to consider the $N(N-1)$ properly located force centers. In order to clarify the physical meaning of the obtained system, let us rewrite its potential as follows

$$
V_{\mathrm{sph}}=\sum_{a} \frac{g}{2 \cos ^{2} \theta_{a}}=\frac{N(N-1) g}{4}+\frac{g}{2} \sum_{a} \tan ^{2} \theta_{a} .
$$

Recall now that

$$
V_{\text {Higgs }}=\frac{\omega^{2} r_{0}^{2} \tan ^{2} \theta}{2}
$$

is the well-known potential of the Higgs oscillator. It generalizes the ordinary oscillator potential for the sphere with the radius $r_{0}$ and inherits all its hidden symmetries [32].

Hence, we obtained the integrable $N(N-1) / 2$-center $N$-dimensional Higgs oscillator of the frequency $\omega=\sqrt{g}$.

The location of the force centers is quite rigid and deserves to be considered in more details. Note that the Higgs oscillator has been invented about thirty years ago and has been studied the hundreds of papers so far (see, e.g. 33. and refs therein). Nevertheless, its anisotropic version was found quite recently [34], whereas the two-center version is not known yet, up to our knowledge.

\section{THREE-PARTICLE CASE: CIRCLE}

The simplest system is the angular part of three-particle model considered in the pioneering paper by Calogero [1]. Actually, this system was considered in the middle of XIX by Jacobi [18] (see also [35]). For $N=3$, we get a particle on circle $S^{1}$ with three force centers defined by the unit vectors $\mathbf{b}^{12}, \mathbf{b}^{23}$ and $\mathbf{b}^{13}$. The angles between them are equal to $\pi / 3$ and $2 \pi / 3$ (Fig. 1):

$$
\cos \alpha_{12,13}=\cos \alpha_{13,23}=1 / 2, \quad \cos \alpha_{12,23}=-1 / 2 .
$$

The above vectors make up the set of positive roots of $A_{2} \equiv s u(3)$ Lie algebra. Completing them by the oppositely directed vectors corresponding to the negative roots, we obtain a system with six force centers. The angular part of the Hamiltonian

$$
\mathcal{I}=\frac{p_{\varphi}^{2}}{2}+\frac{g}{2 \cos ^{2} \varphi}+\frac{g}{2 \cos ^{2}(\varphi+\pi / 3)}+\frac{g}{2 \cos ^{2}(\varphi-\pi / 3)}=\frac{p_{\varphi}^{2}}{2}+\frac{9 g}{1+\cos 6 \varphi}
$$


coincides with (2). It is invariant under the rotation on $\pi / 3$ and the reflection $\varphi \rightarrow-\varphi$, which generate the symmetry group $D_{6} \equiv S_{3} \otimes Z_{2}$ of the hexagon (Fig. 1). Here $S_{3}$ is the symmetric group of three-particle permutations, which $\mathcal{I}$ inherits from the original Calogero Hamiltonian (1). The $Z_{2}$-symmetry corresponds to the reflection-invariance $x_{i} \rightarrow-x_{i}$ of (11). The integrability of this system is obvious. Note that the splitting of the three-particle Calogero Hamiltonian on the angular and radial parts has been used in Ref. 31 for the detailed study of the quantization.

Let us briefly discuss the relation of the system on circle with the superintegrability of three-particle Calogero model. Note that its superintegrability was studied in detail (see [30] and refs. therein). In the center-of-mass system, three from the five functionally independent constants of motion of the original Calogero system survive. Namely, the Hamiltoinian of the two-particle system $\tilde{\mathcal{H}}=p_{r}^{2} / 2+\mathcal{I} / r^{2}$ and its constant of motion

$$
\mathcal{F}=\left(p_{r}^{2}-\frac{6 \mathcal{I}}{r^{2}}\right) p_{r} \sin 3 \varphi+\left(3 p_{r}^{2}-\frac{2 \mathcal{I}}{r^{2}}\right) \frac{p_{\varphi} \cos 3 \varphi}{r}
$$

are reduced from the second and third order (on momentum) Liouville constants of motion. Similarly, the third conserved quantity

$$
\mathcal{K}=\left(p_{r}^{2}-\frac{6 \mathcal{I}}{r^{2}}\right) p_{r} p_{\varphi} \cos 3 \varphi-\left(3 p_{r}^{2}-\frac{2 \mathcal{I}}{r^{2}}\right) \frac{2 \mathcal{I} \sin 3 \varphi}{r}
$$

is inherited from the additional third order constant of motion of three-particle Calogero system. The integrals $\tilde{\mathcal{H}}$, $\mathcal{F}$, and $\mathcal{K}$ are functionally independent. We have expressed them in terms of the angular part of the Hamiltonian (8), which also conserves. Its Poisson bracket action maps the Liouville integral to the additional one:

$$
\{\mathcal{I}, \mathcal{F}\}=3 \mathcal{K}, \quad\{\mathcal{I}, \mathcal{K}\}=-6 \mathcal{I} \mathcal{F}
$$

The four quantities $\tilde{\mathcal{H}}, \mathcal{I}, \mathcal{F}$, and $\mathcal{K}$ form an overcompleted set of constants of motion. They and subjected to the algebraic relation

$$
\mathcal{K}^{2}+2 \mathcal{I} \mathcal{F}^{2}=8 \tilde{\mathcal{H}}^{3}(2 \mathcal{I}-9 g), \quad \text { or } \quad \quad \mathcal{I}=\frac{\mathcal{K}^{2}+72 g \tilde{\mathcal{H}}^{3}}{16 \tilde{\mathcal{H}}^{3}-2 \mathcal{F}^{2}}
$$

Hence, one can choose $\tilde{\mathcal{H}}, \mathcal{I}$, and $\mathcal{F}$ as a complete set of functionally independent conserved quantities. The first two of them are quadratic on momenta, which ensures the separation of variables in the system.

It is easy to verify that the Poisson brackets (11) are in consistency with the relation (12). Finally, using (12) and the first equation in (11), we obtain the Poisson bracket between two third-order integrals:

$$
\{\mathcal{K}, \mathcal{F}\}=3\left(8 \tilde{\mathcal{H}}^{3}-\mathcal{F}^{2}\right)=3 \frac{\mathcal{K}^{2}+9 g \mathcal{F}^{2}}{2 \mathcal{I}-9 g} .
$$

\section{FOUR-PARTICLE SYSTEM: SPHERE}

In the four-particle case, everything becomes much more complicated. In the same way, we obtain a system on the sphere with six force centers defined by the unit vectors $\mathbf{b}^{a}$ with the following Cartesian coordinates of the ambient $\mathbb{R}^{3}$ space:

$$
\begin{aligned}
& \mathbf{b}^{12}=\left(\sqrt{\frac{2}{3}},-\frac{1}{\sqrt{3}}, 0\right), \quad \mathbf{b}^{13}=\left(\sqrt{\frac{2}{3}}, \frac{1}{2 \sqrt{3}},-\frac{1}{2}\right), \quad \mathbf{b}^{14}=\left(\sqrt{\frac{2}{3}}, \frac{1}{2 \sqrt{3}}, \frac{1}{2}\right), \\
& \mathbf{b}^{23}=\left(0, \frac{\sqrt{3}}{2},-\frac{1}{2}\right), \quad \mathbf{b}^{24}=\left(0, \frac{\sqrt{3}}{2}, \frac{1}{2}\right), \quad \mathbf{b}^{34}=(0,0,1) .
\end{aligned}
$$

The vertexes of $\mathbf{b}^{i j}$ and their opposite vectors form an Archimedean solid called cuboctahedron (Fig 2). This polyhedron, like cube, has the octahedral symmetry $O_{h} \equiv S_{4} \otimes Z_{2}$ of order 48 . Here $S_{4}$ is the symmetric group of four-particle permutations, which preserve the original Calogero Hamiltonian (11). Note that $S_{4}$ is isomorphic to the Weyl group of $A_{3}$ Lie algebra and preserves the orientation of cuboctahedron. The $Z_{2}$ symmetry corresponds to the reflection $x_{i} \rightarrow-x_{i}$ of all four coordinates. In Lie algebraic description, it corresponds to the reflection symmetry of $A_{3}$ Dynkin diagram. 
Note that the vectors $\mathbf{b}^{23}, \mathbf{b}^{34}$ and $\mathbf{b}^{24}$ belong to the "equatorial" plane, the angles between them are equal to $\pi / 3$ and $2 \pi / 3$. Their vertexes and the vertexes of the opposite vectors form an hexagon (Fig. 2). This is precisely the same picture as in the three-particle Calogero model (see Fig. 1). The endpoints of the vectors $\mathbf{b}^{12}, \mathbf{b}^{13}, \mathbf{b}^{14}$ are located on a plane parallel to the equatorial one (Fig. 21). The distance between both planes is $\sqrt{2 / 3}$. They form the (regular) triangular face of the cuboctahedron, which is shifted by the angle $\pi / 6$ with respect to the triangle $\left(\mathbf{b}^{23}, \mathbf{b}^{34},-\mathbf{b}^{24}\right)$.

Let us choose Cartesian coordinates with the first axis directed along $\mathbf{b}^{13}$ while the second one belonging to the plane formed by $\mathbf{b}^{12}$ and $\mathbf{b}^{13}$. The frame directions then are orthogonal to the triangles of the cuboctahedron (Fig. 22). In the respective spherical coordinates, the angular part of the Hamiltonian reads:

$$
\mathcal{I}=\frac{p_{\theta}^{2}}{2}+\frac{p_{\varphi}^{2}}{2 \sin ^{2} \theta}+\frac{9 g\left(8-\tan ^{2} \theta\right)^{2}}{2\left(3 \tan ^{2} \theta-8+\tan ^{3} \theta \cos 3 \varphi\right)^{2}}+\frac{12 g}{3 \tan ^{2} \theta-8+\tan ^{3} \theta \cos 3 \varphi}+\frac{9 g}{4 \sin ^{2} \theta(1+\cos 6 \varphi)} .
$$

The invariance under $Z_{3}$ group of the rotation on $2 \pi / 3$ along the third axis is apparent.

The potential (14) is really horrible. It is difficult to believe, that the system with such potential could be integrable, or could admit a separation of variables. However, the Hamiltonian can be represented in a much simpler form. Indeed, there are three pairs of the orthogonal vectors $\mathbf{b}^{12} \cdot \mathbf{b}^{34}=\mathbf{b}^{13} \cdot \mathbf{b}^{24}=\mathbf{b}^{14} \cdot \mathbf{b}^{23}=0$. Taking the vector products of these pairs, one can find out that they form an orthogonal frame:

$$
\mathbf{a}_{1} \equiv \mathbf{b}^{12} \times \mathbf{b}^{34}, \quad \mathbf{a}_{2} \equiv \mathbf{b}^{13} \times \mathbf{b}^{24}, \quad \mathbf{a}_{3} \equiv \mathbf{b}^{14} \times \mathbf{b}^{23}: \quad \mathbf{a}_{i} \cdot \mathbf{a}_{j}=\delta_{i j} .
$$

The vectors $\mathbf{a}_{i}$ are normal to the squares of the cuboctahedron (Fig 21). In this coordinate system, the Hamiltonian (4) looks like

$$
\tilde{\mathcal{H}}=\sum_{i=1}^{3} \frac{p_{i}^{2}}{2}+\sum_{1 \leq i<j \leq 3}\left(\frac{g}{\left(u_{i}-u_{j}\right)^{2}}+\frac{g}{\left(u_{i}+u_{j}\right)^{2}}\right), \quad\left\{p_{i}, u_{j}\right\}=\delta_{i j},
$$

where, again, we keep the old notations for the new momenta. This is the three-particle $D_{3}$ Calogero model [20]. However, this is an expected result, since the diagrams $D_{3}$ and $A_{3}$ coincide and define the same algebra (in the Dynkin classification, $D_{n}$ is defined for $n \geq 4$ ).

The angular part of this Hamiltonian has the following form:

$$
\mathcal{I}=\frac{p_{\theta}^{2}}{2}+\frac{p_{\varphi}^{2}}{\sin ^{2} \theta}+\frac{4 g}{\sin ^{2} \theta}\left[\frac{1}{1+\cos 4 \varphi}+\frac{k-6}{k-8+8 / k-k \cos 4 \varphi}+\frac{4(k-16+16 / k)}{(k-8+8 / k-k \cos 4 \varphi)^{2}}\right],
$$

where

$$
k=\tan ^{2} \theta=\frac{1-\cos 2 \theta}{1+\cos 2 \theta} .
$$

In these new spherical coordinates, the invariance under $Z_{4}$ rotations $\varphi \rightarrow \varphi+\pi / 4$ and spatial reflections $\theta \rightarrow \pi-\theta$, which are a subgroup in $O_{h}$, is transparent.

As was explained above and showed explicitly for three-particle system, $\mathcal{I}$ can be expressed in terms of the five integrals of the maximally superintegrable Hamiltonian (16). It seems that the two additional integrals of $\tilde{\mathcal{H}}$ can be obtained from the Liouville integrals by Poisson bracket action with $\mathcal{I}$ like in the three-particle case (11).

Since the spherical system (17) was obtained from the Calogero model, it is also integrable. Its constants of motion can be obtained from those of the original model.

\section{SUMMARY AND DISCUSSION}

In conclusion, let us emphasize the main results of the current article.

We have found that the angular part of $N$-particle rational Calogero model (in the center-of-mass system) gives rise to the $N(N-1) / 2$-center hyperspherical (Higgs) oscillator. Its relations with the superintegrability of the Calogero system has been discussed briefly.

For the three-particle system, we have related the angular part of the Calogero Hamiltonian (which is quadratic on momentum) with the higher order constants of motion.

For the four-particle Calogero model, the force centers are located at the vertexes of the Archimedean solid cuboctahedron. 
We are planning in future studies to investigate the reflection of the superintegrability of the rational four-particle Calogero model on the properties of corresponding spherical system and, presumably, to extend this investigation to the case of $N>4$ particles. In particular, this would help us to answer whether the the Hamiltonian (17) admits a separation of variables. We expect that the Poisson bracket of the angular part with the Liouville constants of motion will generate the additional serie of conserved quantities, which are responsible for the superintegrability.

Another task is to extract and study the angular parts of Calogero models associated with other Lie algebras. It is obvious that they are also connected with a multi-center spherical integrable system related with (high-dimensional) polyhedra.

It is clear that the proposed construction is applicable for the quantum spin-Calogero model too. In that case the force centers of the angular part will be equipped by the spin- $1 / 2$ exchange interactions [36]. In this context, the relation of the four-particle system with the cuboctahedron becomes important. We expect that using the freezing trick [37], one can obtain an integrable spin lattice system on cuboctahedron. Note that recently the Heisenberg spin systems on large magnetic molecules has been investigated intensively [38]. In particular, cuboctahedric molecular magnets with nearest-neighbor Heisenberg interaction has been obtained experimentally and studied theoretically [39]. It will be interesting to consider from this viewpoint $N=2$ supersymmetric Calogero system [40]. The construction of the $\mathcal{N}=4$ supersymmetric counterpart of the suggested system and the study of its integrability is also important. For the cuboctahedric system, the solution of this task is more or less obvious: we just need to check whether is it possible to represent the Hamiltonian (14) or (17) in the form [41]

$$
\mathcal{I}=\frac{1}{g(z, \bar{z})}\left(\pi \bar{\pi}+\frac{F(z) \bar{F}(\bar{z})}{(1+\lambda(z) \bar{\lambda}(\bar{z}))^{2}}\right), \quad\{\pi, z\}=1, \quad \text { where } \quad \bar{\lambda}^{\prime} F=-\lambda \bar{F} .
$$

In this respect, the recent paper [42], where the supersymmetric Calogero model was related with the nontrivial deformation of spin-Calogero model, deserves to be mentioned.

\section{Acknowledgments}

We are grateful to Sergey Krivonos for the discussions on three-particle Calogero system, which prompted us to this study, and to Vadim Ohanyan and Olaf Lechtenfeld for the interest in work and useful comments. The work was supported by grants NFSAT-CRDF UC-06/07, INTAS-05-7928 (T.H. \& A.N.), ANSEF-1386PS (T.H.), ANSEF1730PS (A.N. \& V.Y.), and by the Artsakh Ministry of Education and Science (A.N. \& T.H.).

[1] F. Calogero, J. Math. Phys. 10 (1969) 2191.

[2] F. Calogero, J. Math. Phys. 12 (1971) 419.

[3] J. Moser, Adv. Math. 16 (1975) 197.

[4] A. P. Polychronakos, J. Phys. A 39 (2006) 12793.

[5] J. A. Minahan and A. P. Polychronakos, Phys. Lett. B 326 (1994) 288.

A. Gorsky and N. Nekrasov, Nucl. Phys. B 414 (1994) 213.

[6] G. W. Gibbons and P. K. Townsend, Phys. Lett. B 454 (1999) 187.

[7] A. P. Polychronakos, Phys. Rev. Lett. 74 (1995) 5153.

[8] A. P. Polychronakos, Nucl. Phys. B 324 (1989) 597; see also A. P. Polychronakos, "Generalized statistics in one dimension", Les Houches 1998 lectures arXiv:hep-th/9902157.

[9] R. B. Laughlin, Phys. Rev. Lett. 50 (1983) 1395.

[10] H. Azuma and S. Iso, Phys. Lett. B 331 (1994) 107.

[11] B. Sutherland, Phys. Rev. A 4 (1971) 2019; ibid. 5 (1972) 1372.

[12] F. D. M. Haldane. Phys. Rev. Lett. 60 (1988) 635; 66 (1991) 1529.

B. S. Shastry. Phys. Rev. Lett. 60 (1988) 639.

[13] A. G. Abanov and P. B. Wiegmann, Phys. Rev. Lett 95, 076402 (2005).

[14] A. Jevicki, H. Levine, Phys. Rev. Lett. 44 (1980) 1443.

A. P. Polychronakos, Phys. Lett. B 266 (1991) 29.

[15] K. Hikami and M. Wadati, Phys. Rev. Lett. 73 (1994) 1191.

[16] D. Bernard, M. Gaudin, F. D. M. Haldane, and V. Pasquier, J. Phys. A 26, 5219 (1993).

[17] F. J. Dyson, J. Math. Phys. 3 (1962) 140, 157, 166.

[18] C. Jacobi, GesammelteWerke, Berlin, 4 (1866) 533.

[19] J. Wolfes, J. Math. Phys. 15 (1974) 1420;

F. Calogero and C. Marchioro, J. Math. Phys. 15 (1974) 1425;

M. A. Olshanetsky and A. M. Perelomov, Lett. Math. Phys. 2 (1977) 7. 
[20] M. Olshanetsky and A. Perelomov, Phys. Rept. 71 (1981) 313; ibid. 94 (1983) 313.

[21] R. Sasaki and K. Takasaki, J. Math. Phys. 47 (2006) 01270.

[22] N. Gurappa and P. K. Panigrahi, Phys. Rev. B 59 (1999) R2490;

P. Ghosh, Nucl. Phys. B 595 (2001) 519;

T. Brzeziński, C. Gonera, and P. Maślanka, Phys. Lett. A 254 (1999) 185.

[23] A. Galajinsky, O. Lechtenfeld, and K. Polovnikov, Phys. Lett. B 643 (2006) 221.

[24] D. Freedman and P. Mende, Nucl. Phys. B 344 (1990) 317.

[25] A. Galajinsky, O. Lechtenfeld, and K. Polovnikov, JHEP 0711 (2007) 008; N=4 mechanics, WDVV equations and roots, arXiv:0802.4386.

O. Lechtenfeld, WDVV solutions from orthocentric polytopes and Veselov systems, arXiv:0805.3245.

[26] T. Hakobyan and A. Nersessian, Phys. Lett. A 373 (2009) 1001.

[27] S. Bellucci, S. Krivonos, and A. Sutulin, Nucl. Phys. B 805 (2008) 24.

[28] S. Wojciechowski, Phys. Lett. A 95 (1983) 279.

[29] C. Conera, Phys. Lett. A 237 (1998) 365;

M. F. Ranada, J. Math. Phys. 40 (1999) 236.

[30] S. Benenti, C. Chanu, and G. Rastelli, J. Math. Phys. 41 (2000) 4654;

J. T. Harwood, R. G. McLenaghan, and R. G. Smirnov, Comm. Math. Phys. 259 (2005) 679;

R. G. Smirnov, P. Winternitz, J. Math. Phys. 47 (2006) 093505.

[31] L. Feher, I. Tsutsui, and T. Fulop, Nucl. Phys. B 715 (2005) 713.

[32] P. W. Higgs, J. Phys. A 12 (1979) 309;

H. I. Leemon, J. Phys. A 12 (1979) 489.

[33] D. Bonatos, C. Daskaloyanis, and K. Kokkatos, Phys. Rev. A 50 (1994) 3700;

E. Kalnins, W. Miller, and G. Pogosyan, J. Math. Phys. 41 (2000) 2629;

A. Nersessian and G. Pogosyan, Phys. Rev A 63 (2001) 20103(R);

A. Ballesteros, F. J. Herranz, and O. Ragnisco, J. Phys. A 38 (2005) 7129;

A. Ballesteros and F. J. Herranz, J. Phys. A 40 (2007) F51;

J. F. Carinena, M. F. Ranada, and M. Santander, Ann. Phys. 322 (2007) 2249.

[34] A. Nersessian and V. Yeghikyan, J. Phys. A 41 (2008) 155203.

[35] A. M. Perelomov, Integrable systems of classical mechanics and Lie algebras, Nauka Publ., Moscow, 1989.

[36] J. A. Minahan and A. P. Polychronakos, Phys. Lett. B 302 (1993) 265.

[37] A. P. Polychronakos, Phys. Rev. Lett. 70 (1993) 2329.

[38] J. Schnack, Molecular Magnetism, in Quantum Magnetism, Lecture Notes in Physics 645 (2004) 155 (Springer-Verlag, Berlin).

[39] R. Schmidt, J. Richter, and J. Schnack, J. Magn. Magn. Mater. 295 (2005) 164;

J. Schnack, J. Richter, and R. Schmidt, Phys. Rev. B 76 (2007) 054413;

I. Rousochatzakis, A. M. Läuchli, and F. Mila, Phys. Rev. B 77 (2008) 094420.

[40] D. Z. Freedman and P. F. Mende, Nucl. Phys. B 344 (1990) 317.

[41] S. Bellucci et al., Phys. Lett. B 616 (2005), 228;

S. Bellucci and A. Nersessian, Phys. Rev. D 73 (2006) 107701.

[42] S. Fedoruk, E. Ivanov, and O. Lechtenfeld, Supersymmetric Calogero models by gauging, arXiv:0812.4276 